\title{
Recording of Time-Varying Back-Pain Data: A Wireless Solution
}

\author{
Tacha Serif and Gheorghita Ghinea, Member, IEEE
}

\begin{abstract}
Chronic back pain is a debilitating experience for a considerable proportion of the adult population, with a significant impact on countries' economies and health systems. While there has been increasing anecdotal evidence to support the fact that for certain categories of patients (such as wheelchair users), the back pain experienced is dynamically varying with time, there is a relative scarcity of data to support and document this observation, with consequential impact upon such patients' treatment and care. Part of the reason behind this state of affairs is the relative difficulty in gathering pain measurements at precisely defined moments in time. In this paper, we describe a wireless-enabled solution that collects both questionnaire and diagrammatic, visual-based data, via a pain drawing, which overcomes such limitations, enabling seamless data collection and its upload to a hospital server using existing wireless fidelity technology. Results show that it is generally perceived to be an easy-to-use and convenient solution to the challenges of anywhere/anytime data collection.
\end{abstract}

Index Terms - Back pain, pain drawings, ubiquitous data collection, visualization.

\section{INTRODUCTION}

$\mathbf{T}$ ELEMEDICINE refers to the use of telecommunication technology for medical diagnosis, treatment, and patient care [1]. Recent technological advances have enabled the introduction of a broad range of telemedicine applications, such as teleradiology [2]-[4], teleconsultancy [5], telesurgery [6], remote-patient monitoring [7]-[9], and health-care management [10].

The integrated use of telecommunications and information technology in the health sector, however, leads to new challenges in organizing, storing, transmitting, and presenting health information in both a timely and efficient manner for effective health-related decision making. Innovations range from routine hospital information systems [11] to sophisticated artificial intelligence (AI)-based clinical decision-support systems [12]-[15].

Moreover, in today's information-intensive society, consumers of health care want to be better informed of their health options and are, therefore, demanding easy access to relevant health information. Simultaneously, clinicians are eager to exploit advances in telecommunication technology in order to put in practice new methods of data gathering and patient monitoring. While the use of the Internet in this respect is

Manuscript received June 17, 2004; revised October 28, 2004.

The authors are with the School of Information Systems, Computing and Mathematics, Brunel University, Uxbridge, Middlesex UB8 3PH, U.K. (e-mail: tacha.serif@brunel.ac.uk; e-mail: george.ghinea@brunel.ac.uk).

Digital Object Identifier 10.1109/TITB.2005.847514 by now traditional [16]-[18], it is only recently that wireless technologies have been harnessed to act as tools coming to the aid of patients and clinicians alike.

To this end, work has focused on patient monitoring systems and context-aware hospitals. Thus, a patient-monitoring system that uses wireless application protocol (WAP)-enabled devices as mobile access terminals is described in [19]. Using this system, authorized users, hospital personnel, and patients' relatives can access a patient's physiological data stored on the hospital's computer. On the other hand, a context-aware hospital mobile prescription system that can identify and react according to the location of tagged items (personal digital assistants (PDAs), beds, hospital trolleys), prescribing the correct medication to patients based on their bed identification number is detailed in [20], while a context-aware messaging system, which can download the appropriate data to a doctor's PDA according to its location was depicted in [21]. From a different perspective, [22] examined the use of small-screened mobile devices for healthcare services, and showed no significant difference between the use of PDAs and laptops when they are used for nursing documentation.

In this paper, we present the implementation and experiences of a wireless-enabled monitoring system for back-pain patients. The motivation behind our work lies in the fact that, while back pain is a worldwide problem with considerable implications on countries' health-care budgets and national economies, there is a relative paucity of tools for the collection and digitization of back-pain data. Moreover, the disabling pain experienced by back-pain sufferers means that in many cases, such data collection cannot take place unless medical personnel is present at the patient's domicile, a situation which, in most cases, is both unrealistic and impractical. The consequence of this state of affairs is that there is underreporting of back-pain data, as well as an almost total lack of available, continuously polled back-pain data, notwithstanding the evidence in support of the fact that, for chronic back-pain sufferers, pain has a time-dependent nature [23], and that this relation is, as of yet, still not completely understood. Accordingly, the structure of this paper is as follows: Section II presents an overview of the area of back pain, while Section III reviews work done on the visualization of back-pain data. Such work provides the foundation for our project, which is described in detail in Section IV. Last, Section V presents the results of an evaluative study of our back-pain tool, while the implications of our work are elaborated upon in Section VI, where conclusions and possibilities for future work are identified. 


\section{BACK PAIN}

\section{A. Review Stage}

Back pain is a worldwide experience. Disabling back pain appears to be a problem for western and industrialized societies, possibly related to the development of welfare states. Thus, according to a Department of Health survey, in Britain, back pain affects $40 \%$ of the adult population, $5 \%$ of which have to take time off to recover [24]. This causes a large strain on the health system, with some $40 \%$ of back-pain sufferers consulting a GP for help, and 10\% seeking alternative medicine therapy [24]. Due to the large number of people affected, back pain alone cost industry 9090 million U.K. pounds in 1997/8, with between 90 and 100 million days of sickness and invalidity benefit paid out per year for back-pain complaints [25]. Back pain is not confined to the U.K. alone, but is a worldwide problem: in the USA, for instance, $19 \%$ of all workers' compensation claims are made with regard to back pain. Although this is a lot less than the percentage of people affected by back pain in the U.K., it should be noted that not all workers in the USA are covered by insurance, and not all workers will make a claim for back pain [26]. Moreover, back pain does not affect solely the adult population: studies across Europe [27] show that back pain is very common in children, with around $50 \%$ experiencing back pain at some time.

Like most types of pain, back pain is difficult to analyze, as the only information that can be used is suggestive descriptions from the patient. However, these patients may have developed psychological and emotional problems, due to having to deal with the pain. Because of these problems, patients can have difficulty describing their pain, which can lead to problems during the treatment. In some patients, the psychological problems may have aided the cause of the back pain, by adding stress to the body, or the stress of the back pain may have caused psychological problems [28]-[34]. It is because of this factor that patients suffering from back pain are usually asked to fill out questionnaires of different types in order to help the medical staff, not only to know where the pain is located, but also to identify the patient's mental state before treatment begins.

In addition, the patient is usually required to mark on a diagram, usually of a human body, where the pain is located, and the type of pain. This type of diagram is known as a "pain drawing" and forms the primary focus of our paper.

\section{PAIN DRAWINGS AND VISUALIZATION}

\section{A. Pain Drawings}

Pain drawings, as depicted in Fig. 1, have been successfully used in pain centers for over 50 years [35], and act as a simple self-assessment technique, originally designed to enable the recording of the spatial location and type of pain that a patient is suffering from [32], [36], [37]. They have a number of advantages, including being economic and simple to complete, and can also be used to monitor change in a patient's pain situation [37]. Although there are a number of problems with the way that patients behave toward the test when filling them out, especially regarding the way that they like to present themselves to medical staff [30], pain drawings have proven
Mark the areas on your body where you feel thase sersations.
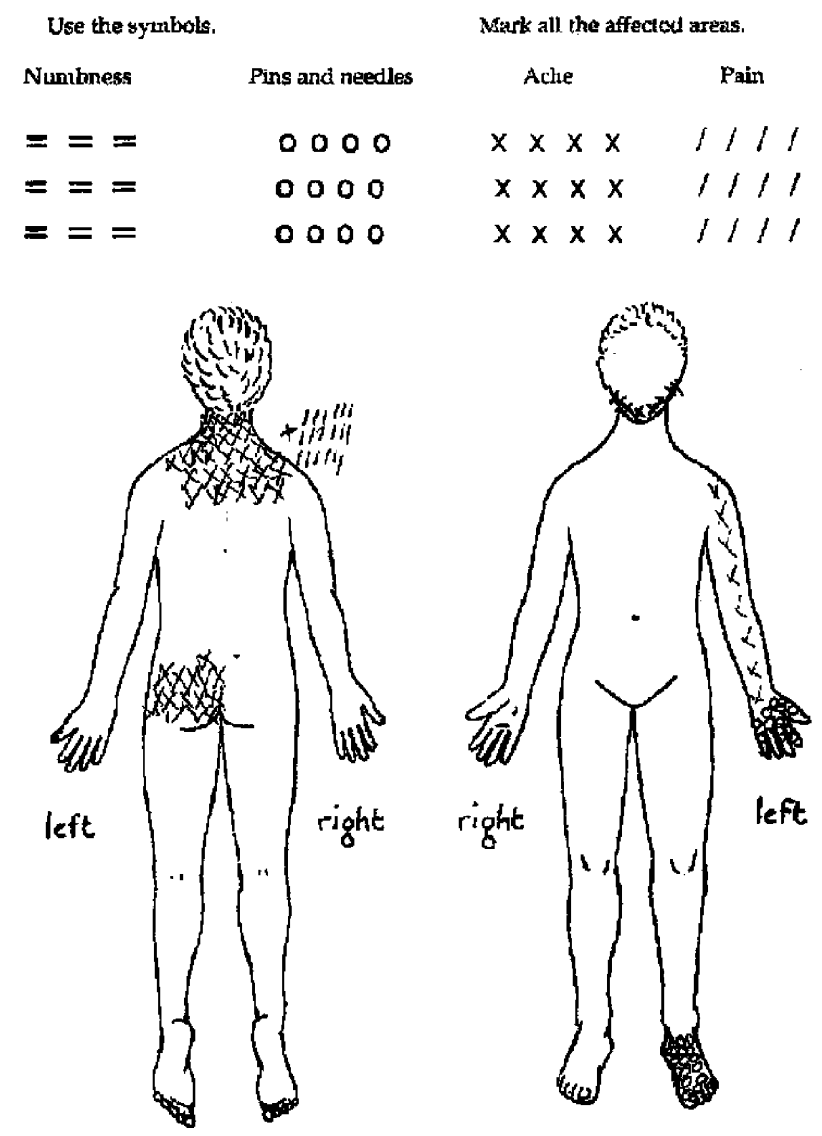

Fig. 1. Example pain drawing.

to be a versatile tool for recording information as diverse as psychological distress, type of pain, and disability [32], [36].

Pain drawings are also flexible enough mechanisms to handle a range of pain descriptors. To this end, Chan et al. [38] use pins and needles, burning, stabbing, and deep ache in their pain drawings, while Hilderbrandt et al. [30] state that they omit the pain qualities because they are not part of the standard pain drawings that they use. On the other hand, Uden et al. [34] use dull, burning, numb, stabbing or cutting, tingling or pins and needles, and cramping in their drawings, while Ohnmeiss uses aching, numbness, pins and needles, burning and stabbing [39].

\section{B. Scoring Pain Drawings}

In order to link the pain drawing to either psychological, emotional, or causes of pain, several scoring systems have been developed and described in the literature. These broadly fall into four categories: grid methods, body-region methods, penalty point system, and visual inspection methods. Whilst the first two record the presence or absence of pain within defined regions, the last two do require subjective interpretation.

With the grid method [40] an overlay of a grid is placed over the pain drawing. The grid is designed so that each cell is approximately the same size. By using the grid, unskilled testers could calculate the amount of surface area that was in pain. 
Body-region methods, on the other hand, break down the surface of the human body in very simple regions, in order to indicate areas that are in pain.

Penalty point systems, such as the one described by Ransford et al. [33], work by awarding points for every unnatural placement of pain on a pain drawing. Different areas and rules are made, so that there is a weighting depending on the irregularities in the drawing. If more points are scored than normal, then that person may have a psychological problem that needs addressing. In this particular case, pain drawings are used not only as a recorder of pain location, but also as an economical psychological screening instrument to see if a patient would react well to back-pain treatment [33]. While traditional psychological screening for back-pain treatment usually entails patients completing costly, time-consuming, and difficult-to-understand questionnaires, by using a penalty-point scoring method, it was found that pain drawings could predict $93 \%$ of the patients that needed further psychological evaluation just by looking at their completed pain drawing, a conclusion later corroborated in [28], [38].

Lastly, visual inspection methods use trained evaluators, who look at the pain drawings, and from their experience, are able to say what they believe to be wrong with the patient, or, indeed, if psychological testing is needed [38]. Most of the methods described can be and are used in practice in conjunction with sensation-type approaches, which allow not only the placement of pain to be noted, but also the particular type of pain encountered. This is done using a key, therefore allowing more information to be collected, and acts as an aid to the clinic as to what the cause of the pain is.

\section{Conclusion}

The consensus of the literature seems to be that the pain diagram is a powerful tool in the role that it is designed for, namely, to record the spatial location and pain type. However, pain drawings are usually stored in a paper format, which allows no further evaluation of the data that is stored upon it, and makes searching through the data a somewhat arduous task. To compound the issue, in the infrequent case when information from the pain drawings is digitized, it invariably results in loss of information, since current systems that are used for analysis of the pain drawings and the associated questionnaires revolve around statistical packages, such as Microsoft Excel and SPSS Inc., incapable of handling diagrammatic data. Thus, although diagrammatic data is collected, it is not used as the key component to the data-analysis tools. This is somewhat a problem, as people will find it easier to show through a diagram the way that they feel, instead of answering closed questions in questionnaires. Such data cannot, therefore, be used to its full potential and, in particular, cannot be used in helping with queries within the dataset.

Last, the paper-based solution of existing methods makes it impractical to record pain variations over time, in spite of the time-dependent nature of pain in chronic sufferers [23]. Fig. 2 shows the pain drawings completed by a wheelchair patient at different times of the same day, clearly highlighting the deterioration of pain, in terms of both diversity and location, as the day wore on. Thus, a potentially valuable source of data is frequently overlooked, to the detriment of patients and clinicians alike.

\section{IMPLEMENTATION}

\section{A. Aim}

In our work, we have sought to alleviate the problems identified above, and have developed a wireless-enabled, ubiquitous solution that uses the pain drawing as an actual user-friendly visual aid to the input and analysis of back-pain datasets. While our solution is generic and applicable to all back-pain sufferers who have access to wireless technology, we have specifically targeted wheelchair users, due to their severe mobility limitations (which might mean that they might not, for instance, easily have access to a desktop-based computer) and their dynamic pain patterns, which are now easily logged by the developed application. In so doing, we specifically address the issue of pain variability in time (Fig. 2), as identified by Gibson and Andrew [23], and our application can thus also be used as a data-gathering tool for this still incompletely understood phenomenon, the solution of which has potentially important implications in the monitoring of the effectiveness of back-pain treatment and medication.

\section{B. Data Collection}

In order to function as an effective data-gathering tool, the developed application, in keeping with previously identified best practice [31]-[34], incorporates a questionnaire complemented by visual input of pain location and type, via a pain drawing.

The questionnaire was elaborated in consultation with clinicians from Northwick Park Hospital (NPH) in London, U.K., and representatives of the U.K. National Forum of Wheelchair User Groups. It is to be remarked that current practice at NPH is that the paper-based pain drawings are not digitized, but physically filed, and that clinicians were thus interested in the recording and digitization of data pertaining to a patient's medical background and capturing the variation of pain patterns with the time of day. On the other hand, the wheelchair users were interested in the usability, flexibility, and privacy aspects of the application. Both stakeholder groups agreed that a wireless solution would be beneficial for the added versatility that it offers.

It was agreed that the pain drawing should incorporate four different pain types, namely, numbness, pins and needles, pain, and ache, and that grid scoring should be used. As opposed to traditional methods [40], in which transparencies of the grid are made, and the drawings are scored by placing the grid over each and counting the number of squares in which the patient indicated symptoms, our approach conceptually slices the body contour into $10 \times 10$-pixel squares (Fig. 3). The advantage brought with this approach was that we were able to code the pain location with its coordinates from an image to a database, and vice versa.

\section{Application Structure}

The underlying structure of our application is based on a three-tier wireless system model (Fig. 4) where the three main 
$10 \mathrm{am}$

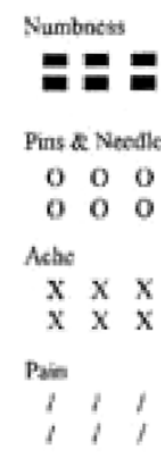

Thak

Neck

Buttocks

legs

Armashoulders

Ficel

Hands

Orarall PainDrecomfort

Pleave tist alher areas

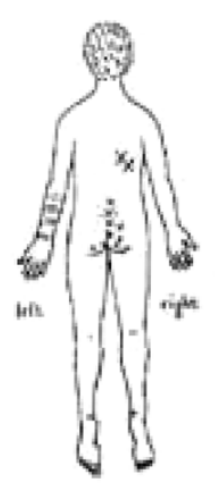

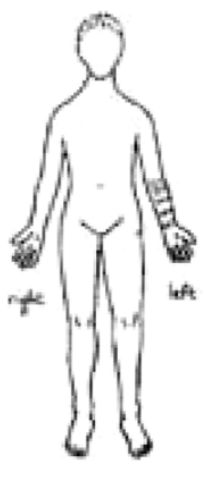

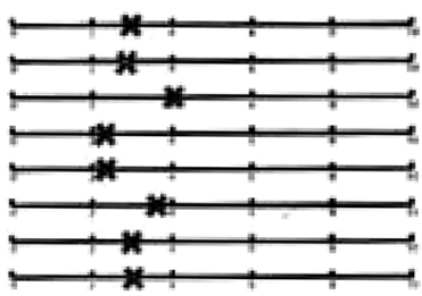

Back

Neck

Butrocks

Legs

Ammsishowder

Feet

Hands

Oreall Paindistomfor

Please list other yeas
$2 \mathrm{pm}$
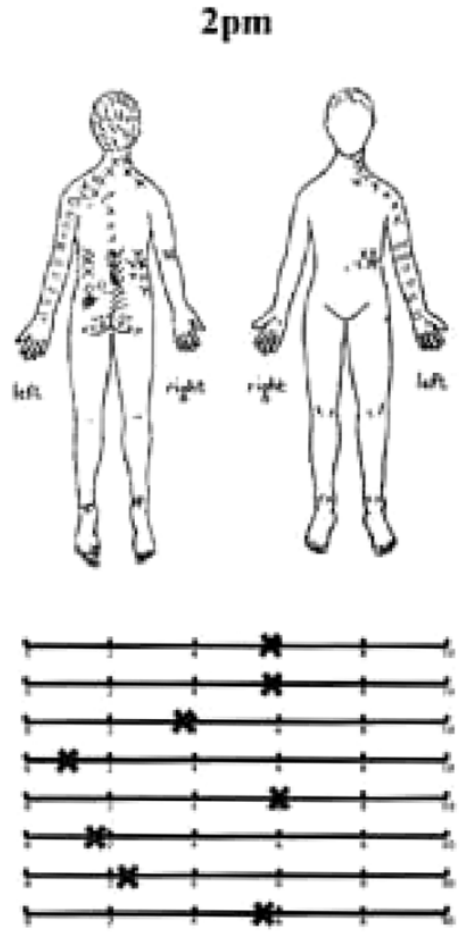

12 Midday
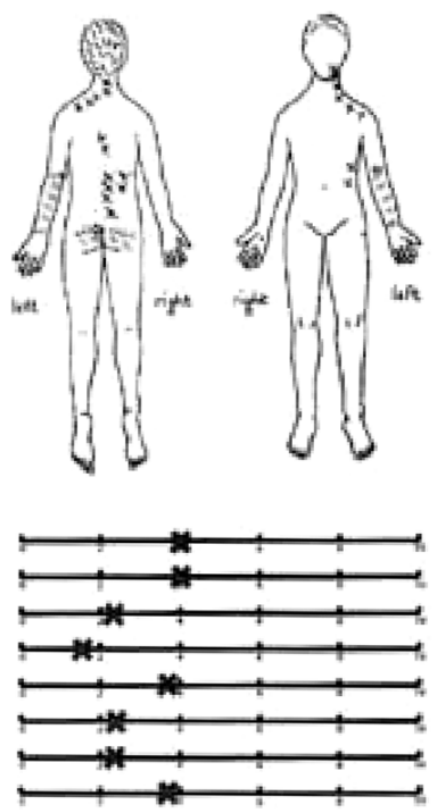

$4 \mathrm{pm}$
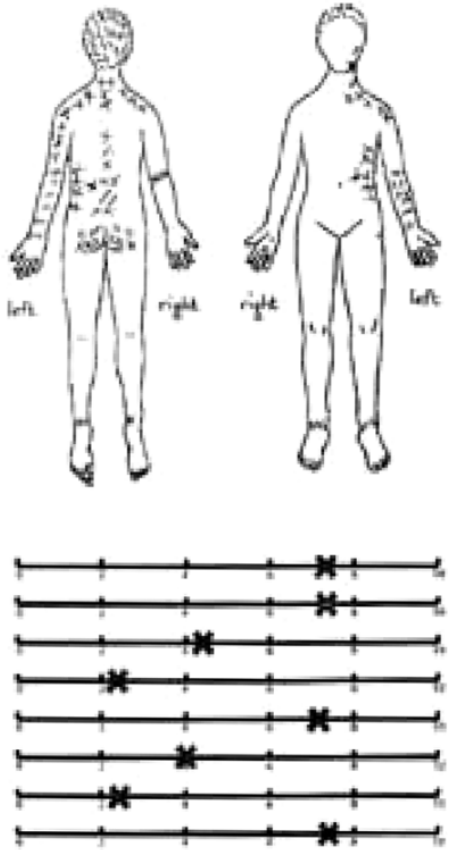

Fig. 2. Time-dependent pain drawings.

components are a mobile, wireless-enabled device, a Web server with scripting capability, and a backend database.

In this model, the patient inputs on a wireless-enabled device (in our case, a PDA) to record pain information. This is done at specific time intervals, as requested by clinicians, and the information is saved to a local database. Whenever the user is within a wireless-enabled zone, s/he then connects to a Web/Database server via a wireless access point, using the Hypertext Transfer Protocol over Secure Socket Layer (HTTPS). Moreover, the connection between the PDA and the wireless access point is itself secured through the use of 128-b wired equivalent privacy (WEP) encryption.

Upon receiving such requests, the server responds back and asks for appropriate authorization. After this has been successfully completed, the data is then uploaded to the hospital server. The clinician then uses his/her computer to logon to the Web server and downloads information regarding any specific patient and their pain pattern from the database for further analysis. 


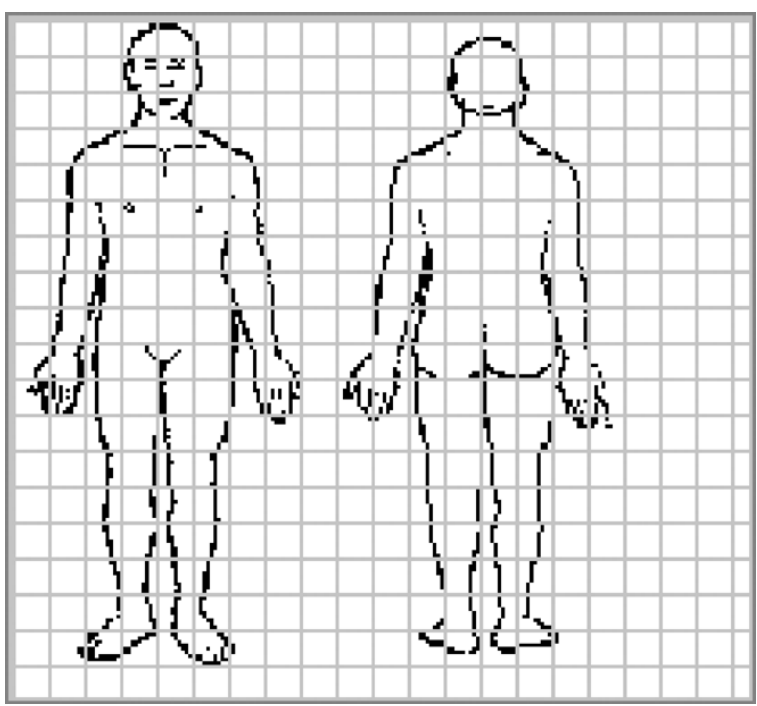

Fig. 3. Virtual grid of the body map.

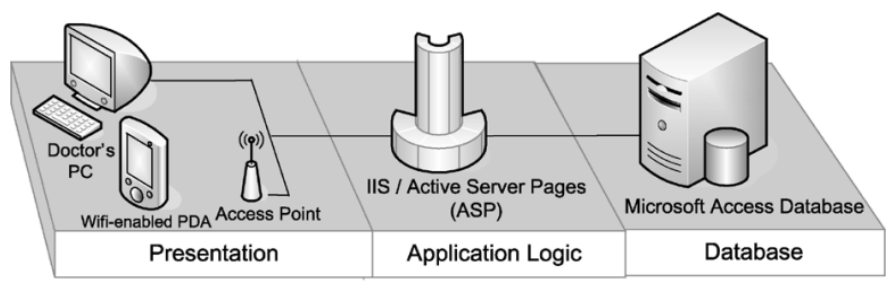

Fig. 4. Wireless system model.

\section{Application Architecture}

The developed back-pain application is designed and implemented using Microsoft Embedded Visual Basic, a language specifically geared to help developers build applications for the next generation of communication and information-access devices running Windows CE.

The system architecture diagram (Fig. 5) shows the main components that make the wireless system model work. Accordingly, the back pain application was implemented on an HP iPAQ 5450 PDA with 16-b touch-sensitive transflective thin film transistor (TFT) liquid crystal display (LCD) that supports 65536 color. The display pixel pitch of the device is $0.24 \mathrm{~mm}$, and its viewable image size is $5.7 \mathrm{~cm}$ wide and $7.7 \mathrm{~cm}$ tall. It runs Microsoft Windows for Pocket PC 2002 (Windows CE) operating system on an Intel $400 \mathrm{Mhz}$ XSCALE processor, and contains $64 \mathrm{MB}$ standard memory, as well as $48 \mathrm{MB}$ internal flash ROM. The Web server was implemented on an Intel Pentium III running at $1 \mathrm{GHz}$, with $512 \mathrm{MB}$ RAM and a 50-GB hard disk. In our work, a $11 \mathrm{Mb} / \mathrm{s}$ D-Link DWL-700 AP wireless access point was used.

The application reads the coordinates of the pain locations from the touch-sensitive screen, and using ADOCE 3.0 (Active Data Objects for CE), connects to a local Microsoft Pocket Access database file. Through this connection, the application saves the pain coordinates and patient questionnaire data to the database. When the user is within wireless Internet coverage, the application uses Winsock CE 3.0 (Windows CE Sockets) to send a connection request to the server. The server is running the Windows 2000 operating system with an Internet information server (IIS) 5.0, which connects to the hospital database through open database connectivity (ODBC). The server hosts a patient database, which is accessed from the web server (IIS 5.0) using ActiveX data objects (ADO).

The doctor's interface is made of dynamically created active server pages (ASP), which can be accessed using any conventional web browser running on a computer connected to the Internet. Thus, after successful authorization, medical personnel can download a particular patient's data to their personal computer. This is achieved through the ASP code dynamically creating a structured query language (SQL) query to the database, the results of which are presented dynamically on the viewed Web page (Fig. 6).

\section{E. Device and User-Interaction Sequence}

The developed system is conceptually made up of two user groups, patient and doctor/clinician, and three devices, namely, a wireless-enabled PDA, a doctor's computer, and a Web/Database server (Fig. 7).

The initial interaction takes place between the doctor and the wireless-enabled PDA device, where the doctor creates a user account for the patient. Following this phase, the patient interacts with the wireless-enabled device, inputting pain data to the device. The device responds to this by creating a graphical representation for every pain point added to the body diagram. After the pain data are saved, whenever a wireless connection is available through an access point, the user can request data transmission to the hospital server. After the connection is established and the patient duly authorized (via a user_name, password tuple), the server can then receive the data that is sent by the wireless-enabled PDA and save it to the hospital database. When this is done, the server initiates connection closure.

After the transfer, the doctor can then interact with his/her desktop computer and, by quoting a patient name, get further detailed information about the respective patient's back-pain progress. Accordingly, the computer interacts with the server and embeds the quote in SQL code, which then is used to query the database. As a result of the query, the database server sends back a response with the requested data to the web server, which then passes the data to the doctor's computer.

\section{FUnCTIONALITY AND EVALUATION}

\section{A. Pilot Evaluation}

The first version of the developed application was given out, together with a brief user manual, to three wheelchair users from the collaborating group for a five-day pilot evaluation. The feedback provided could be broadly categorized into two groups. The first concerned ways through which any potential misunderstandings of the questionnaire content could be clarified. The second grouped issues such as font size (too small in our initial prototype) and color schemes used by the application (which had to take into account users' potential color blindness). The users did not encounter navigation problems, nor were there any problems raised with regard to clarity of the pain diagram, or indeed with the saving and transferring of recorded data. All the 


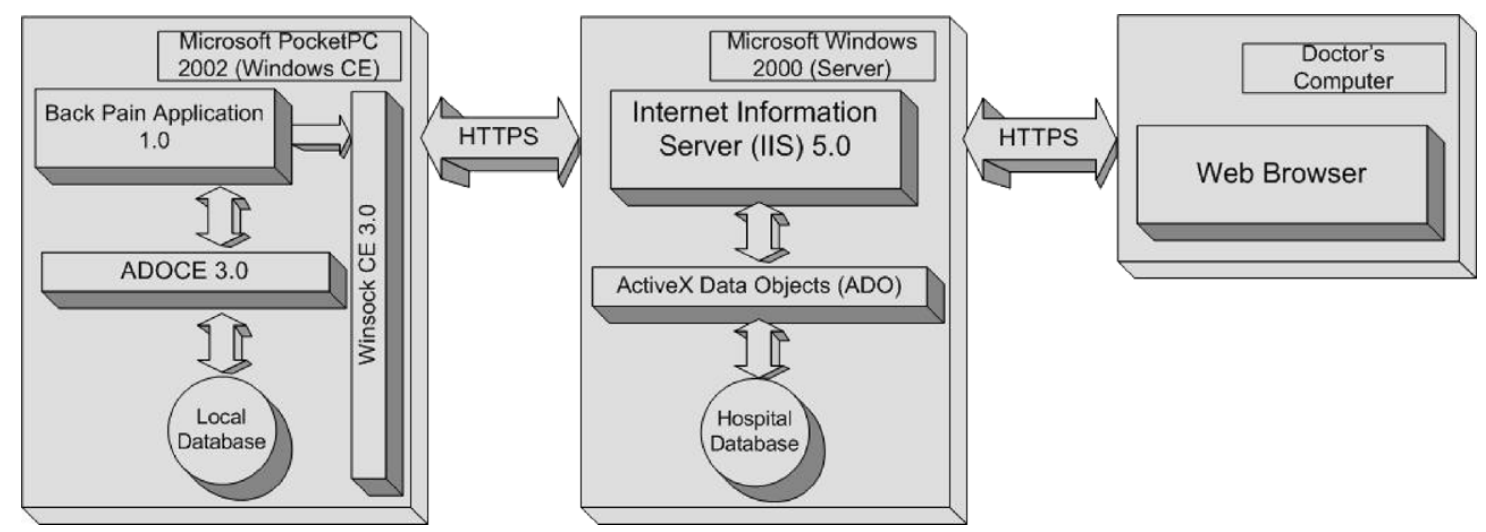

Fig. 5. System architecture diagram.

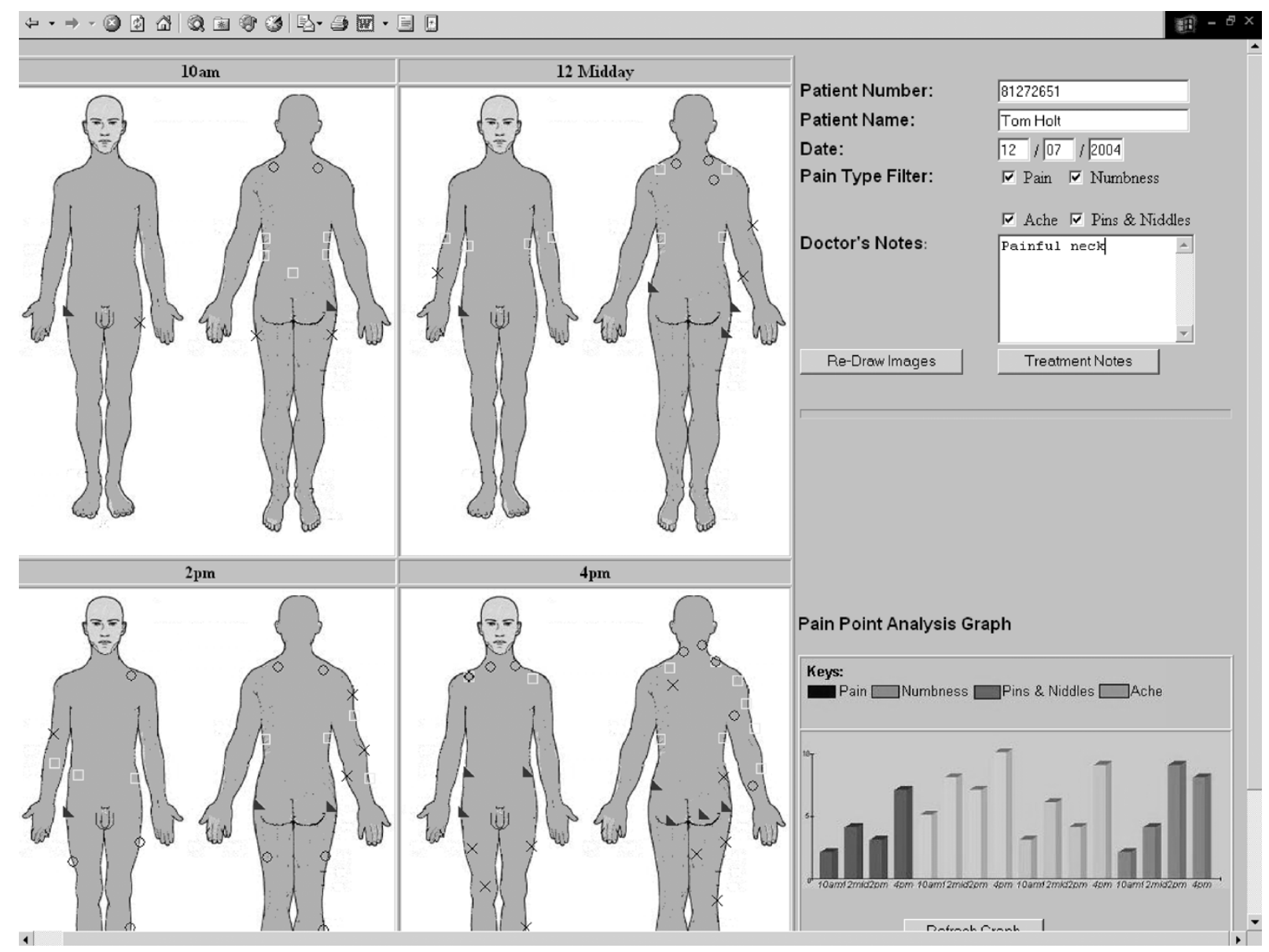

Fig. 6. Doctor's web interface.

concerns identified by the participants of the pilot study were addressed in the subsequent version of our application.

\section{B. Application Walk-Through}

In the developed application, before any pain data can be collected, the clinician first has to create a profile for the particular (in our case, wheelchair) user who is going to log his/her pain information. This the clinician does by tapping on the New Subject button [Fig. 8(a)], the effect of which is to first initialize the database, and then to take the doctor to the user database creation process screen [Fig. 8(b)]. Here the clinician enters information about the patient's personal details, sets up a default password for the user, and inputs background information on the patient such as diagnosis, length of wheelchair use, and wheelchair type (Manual, EPIOC, or EPIC).

The following screen [Fig. 8(c)] requires more specific details about the wheelchair user's pain/discomfort factors. It requires the doctor to detail the factors that increase and, respectively, decrease the pain and discomfort of the wheelchair user, the type of medical treatment received and, if applicable, the type of painkillers prescribed, together with their associated usage 


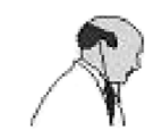

Dogtor
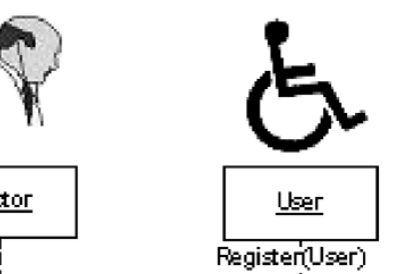
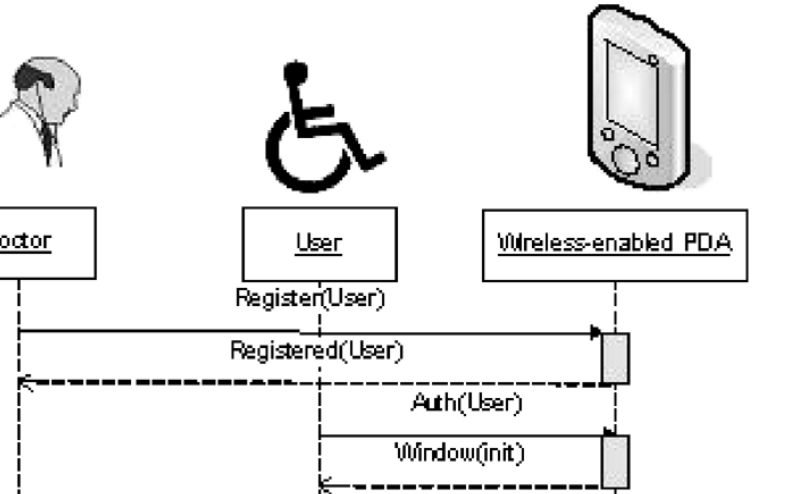

Minreless-enabled PDA
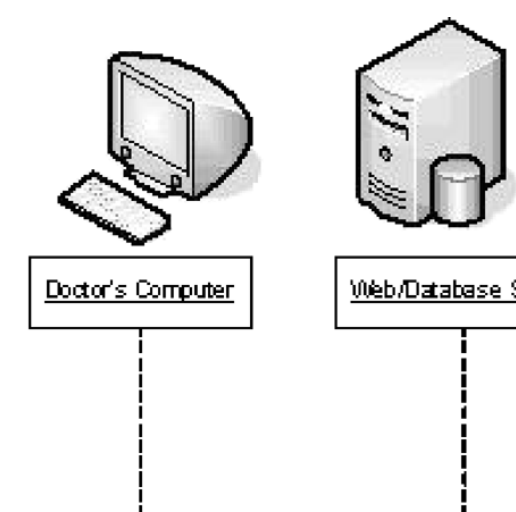

likb, Database Senuer

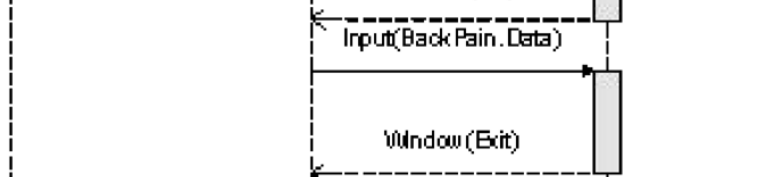




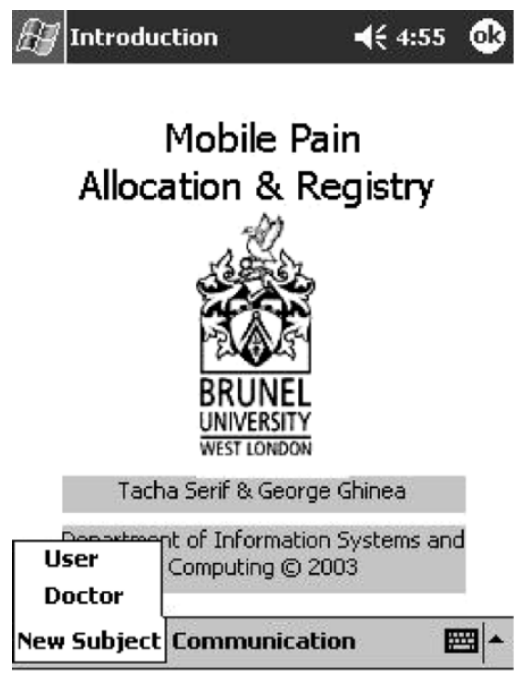

a)

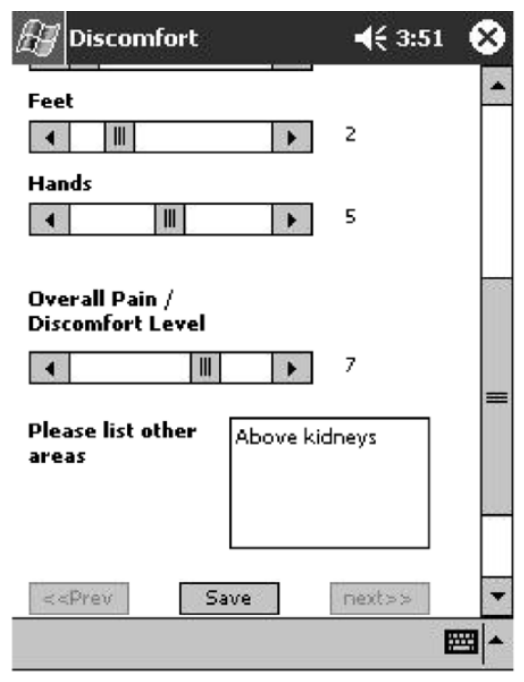

b)

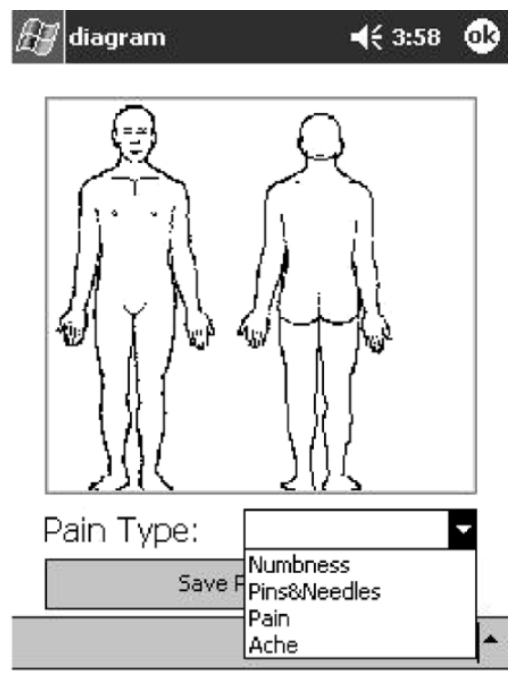

c)

Fig. 9. (a) Patient log-on screen. (b) Discomfort level. (c) Empty diagram.

application page, by selecting the User identity [Fig. 8(a)]. This password can then be changed if the user desires this. The resulting screen [Fig. 8(b)] asks the patient to score his/her discomfort level using a visual analog scale between 1 and 10, as was requested by clinicians. The scale is implemented through the use of scrollbars, which are used to score seven predefined body parts (Back, Neck, Buttocks, Legs, Arms/Shoulders, Feet, and Hands) and give an overall body pain/discomfort level. In addition to these, this screen contains a textbox where the user can add his/her own pain areas and mention how severe this pain or discomfort is. By tapping on the Next button, at the bottom of the screen, the user is taken to a pain diagram where $\mathrm{s} /$ he can select the pain type from the pull-down menu, and specify the pain location by tapping on the body diagram to show exactly where the pain is located. There are four different pain types defined in the menu; these are numbness, pins and needles, pain, and ache [Fig. 9(c)]. In case the user forgets to select the one of the pain types before proceeding to the diagram, the application reminds the user with a message box that a pain type has to be selected first.

Each pain type is represented by a different shape and color: a yellow square for numbness; a black cross for pins and needles; a red triangle for pain; and a blue circle for ache. The colors and symbols were chosen so that potentially a selected location could have all the four pain types displayed concurrently and clearly. Subsequent to pain-type selection, tapping on the screen creates one of the shapes mentioned which symbolizes the pain type and its location on the overall body. The patients can add as many pain symbols as they want to present their pain type and location [Fig. 9(a)]. In case of erroneous pain-type or pain-location selection, the user can delete any symbol from the diagram by selecting the same pain type with the target symbol and then tapping on it. When the user has finished inputting his/her pain information of the diagram, s/he then taps on the Save Pain Points button and saves the pain type and location data, as well as the particular time at which these were gathered, on the local database (Fig. 10).
The data stored on the PDA database can only be sent to the main hospital database while the user is within a wireless Internet area. When this is the case, the user can upload the local PDA database (typical database sizes in our study varied between 180-246 KB for one day's worth of logged back-pain data) to the remote hospital server by tapping on the Соттиnication menu from the introduction screen, and selecting the Upload Data item from the menu [Fig. 10(b)]. Subsequently, the application loads a status screen [Fig. 10(c)], which displays the server name and Internet Protocol (IP) address that the application is connecting to, as well as the authentication details being used. The screen also displays the table name that is being transferred, and a gauge showing the percentage of data transmitted. The user has preemptive capabilities during this process, and can interrupt the transmission any time by tapping on Cancel button at the bottom of the screen. After the transmission is completed, the application reverts to the introductory screen [Fig. 8(a)].

\section{User Evaluation}

The developed application was evaluated with a sample of 50 wheelchair users, members of the U.K. National Forum of Wheelchair User Groups. These were divided into two equalsized groups, a control group, in which participants recorded their pain data on paper using the questionnaire of Fig. 2, and one which used the developed PDA application for data input. Participants were aged between 27-65 years old, each of whom had varying degrees of daily wheelchair use. Each participant was given five days in which to evaluate the application, as well as a short (three-page) user manual, and instructions on which times of the day they should record their pain measurements (every two hours between 10 a.m. and 4 p.m.) and on how to "upload" the data.

At the end of the evaluation period, participants were requested to complete a questionnaire (Table I), in which they recorded their opinions on a Likert scale of 1-7 about the usability and feasibility of using the two ways of recording pain 


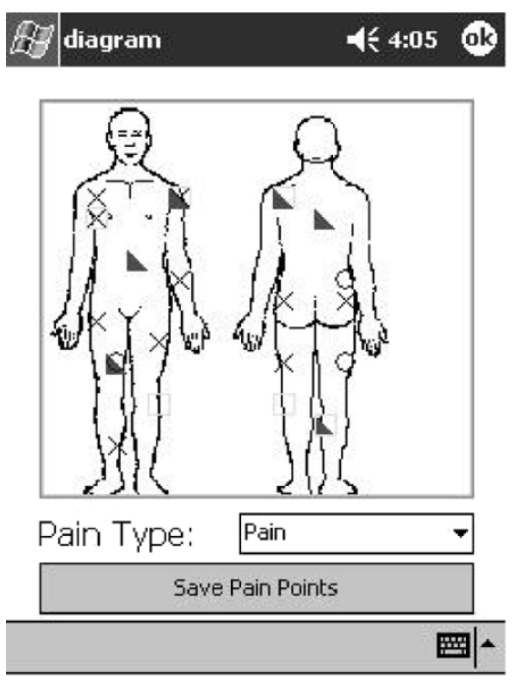

a)

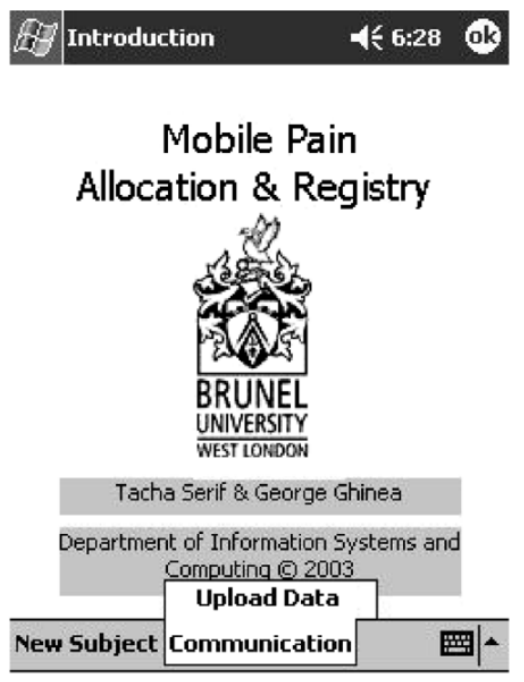

b)

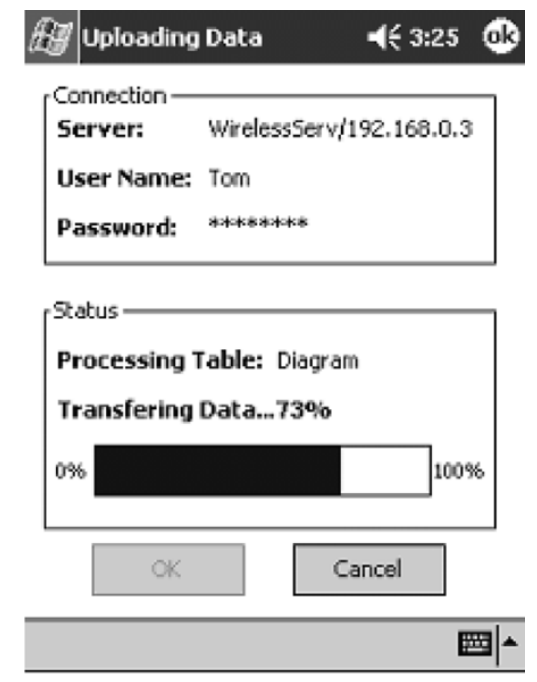

c)

Fig. 10. (a) Diagram with pain points. (b) Communication menu. (c) Upload screen.

TABLE I

EVALUATION QUESTIONNAIRE

It is important to be able to record my pain on a PDA/pain diagram.
It is useful to be able to log pain data across time.
I find the process of inputting pain data on a PDA/pain diagram easy.
Transferring data from PDA/ pain diagram to the main database is easy.

data in practice. Patients were also asked to note any other observations that they might wish to make.

The user manual differed from one group to another-while in the case of the group using the PDA for data input, it focused mainly on explaining the features and functionalities of the PDA application, in the case of the control group, particular attention was given to explaining how the patients should record the location of the pain data in a nongraphical format. This was because the intended output of the two groups was to be the same, namely, a log, both graphical and nongraphical, of the pain location and type across time. While capturing the exact grid coordinates of the pain data is done transparently to the user by the developed PDA software, in the case of the control group, this had to be done by the user. Thus, participants in this group were given a plastic transparent sheet with a grid (similar to Fig. 3), and by overlaying the sheet on top of the completed pain drawings, recorded the $x$ and $y$ coordinates of the particular grid containing the pain indicator, as well as the type of pain itself. Last, participants in this group were told that the last question of the questionnaire addressed this particular bit of the exercise, namely, the transfer of the pain $\operatorname{logs}$ to a paper database, as is the case with current working practice.

Input of data took place mainly at the user's domiciles (or wherever they happened to be when the recording of data had to take place), with no personnel being on hand to offer help in this respect, save for the information contained in the manual. While, in the case of the group using the PDA software, the degree of local connectivity of each patient varied (three of whom had wireless LANs already installed in their homes), they were told to use their own means and resources in order to upload the collected data, once a day, to the hospital server. Although upload times did vary across the cohort (factors such as signal strength, environment, number of users, and data sizes all come into play here), and were on average $47 \mathrm{~s}$ (with a maximum recorded time of $71 \mathrm{~s}$ ), no patient reported any concerns in this respect (Fig. 11). Conversely, in the case of the group using paper-based data input, self-reported times with respect to recording of pain location and type have an average of $267 \mathrm{~s}$. The results of the evaluation are given in Fig. 12.

Our results (Fig. 13) highlighted a general consensus that wheelchair users had in respect to the ability to record pain data on a mobile device being beneficial to their lifestyles (an observation also confirmed through informal and formal written feedback, at the end of the questionnaires). Moreover, running an independent samples t-test on our results revealed that, while in the first three questions of our evaluation, there were no significant differences between a paper-based approach and the PDA one, as far as the last question is concerned, results suggest that user perceptions of the two approaches are radically different $(\mathrm{P}<0.0001)$.

This is generally in line with our expectations. Thus, while opinions about the usefulness of recording pain data and of doing so across time remained roughly the same across both cohorts, we were mildly surprised that while the mean opinion score with respect to ease of data input was higher in the control case than when the PDA was used, the difference was not statistically significant, since we expected participants to have more problems using stylus-based input than the traditional paperbased one (Fig. 11). Nonetheless, the obvious analogies between the two modes of input seem to have overridden our concerns in this respect. Moreover, although some users, especially those suffering from arthritis and/or poorer eyesight, did encounter difficulties in using the relatively small interface of the PDA, overall, the participants agreed that the processes of recording pain data was a relatively easy one and that the ability to record 


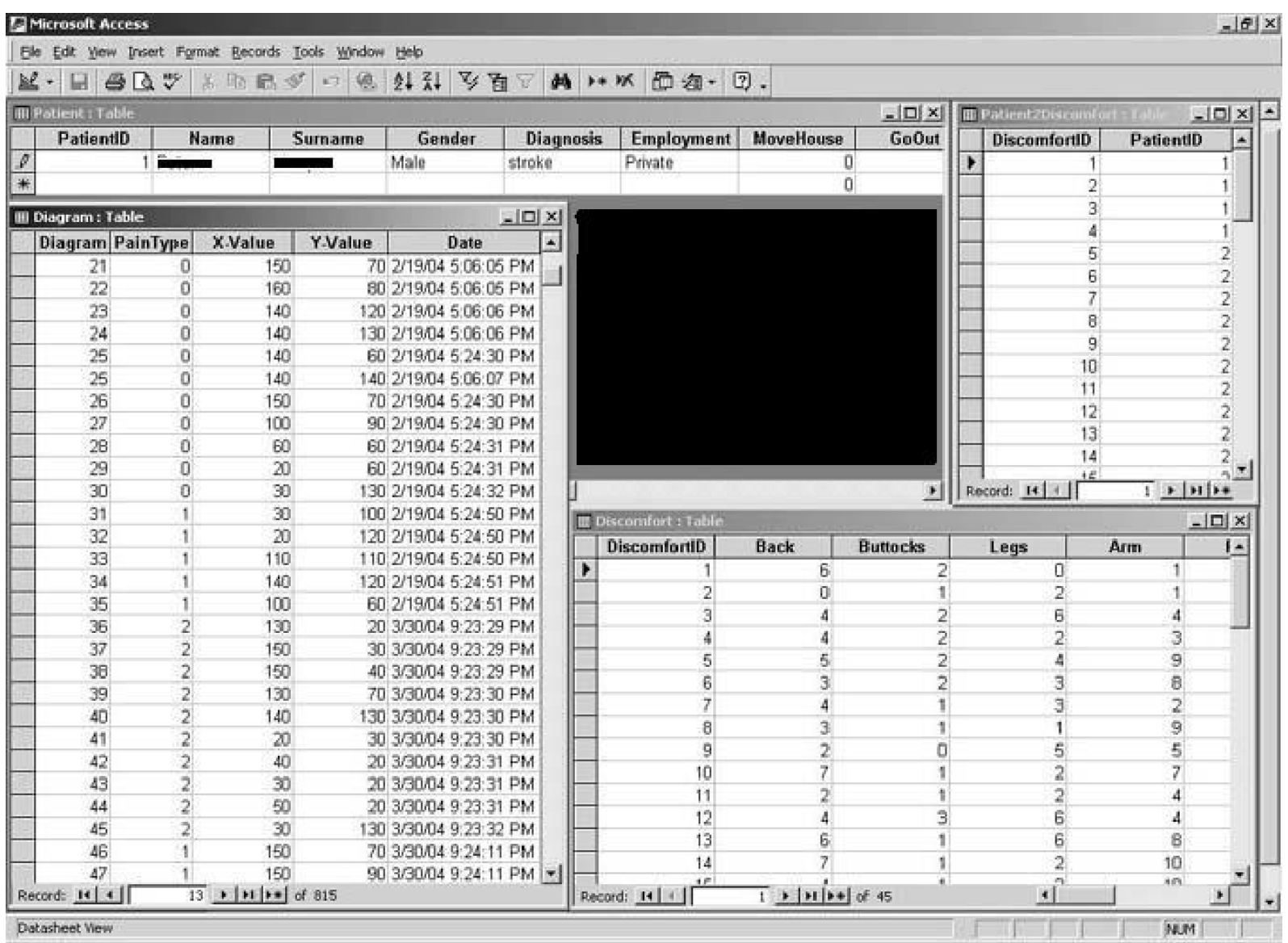

Fig. 11. Snapshot of back-pain database.

data across time, irrespective of the particular location in which users found themselves, was indeed beneficial.

Since the developed PDA-based software transparently records pain location and type to a database, it comes as no surprise that users in the control group, who were asked to manually record this data, had significantly different opinions from this perspective, with this finding highlighting the obvious advantage of digital-based data gathering over a purely paper-based exercise, with limited (if any) possibilities for future data analysis. Last, although participants in the PDA groups did encounter barriers in respect to their attempts to upload data, with some of them using ingenious resources (such as using wireless fidelity (WiFi)-enabled cafes or local shopping malls) to accomplish this task, nonetheless, participants felt generally positive about ubiquitous data collection and transmission capabilities, with the feeling that proliferation of WiFi hotspots would remove such barriers in the future.

\section{Clinical Evaluation}

The doctor view of the application was also evaluated by four clinicians from NPH. They were given seven days to test it and the quality of the data thus provided. At the end of this period, they were asked to complete an open-ended questionnaire requiring them to specify the three most liked and disliked features of the application.
TABLE II

DOCTORS' OPEN-ENDED QUESTIONNAIRE FEEDBACK

\begin{tabular}{|c|c|}
\hline \multicolumn{2}{|l|}{ Likes } \\
\hline More useful in prescribing medication/treatment & 4 \\
\hline More useful in monitoring the effect of treatment & 3 \\
\hline $\begin{array}{l}\text { Beneficial to see how wheelchair pain varies/intensifies } \\
\text { in time }\end{array}$ & 3 \\
\hline $\begin{array}{l}\text { Good for seeing potential pain differentials depending on } \\
\text { wheelchair type }\end{array}$ & 1 \\
\hline Easy to share and transmit data & 1 \\
\hline \multicolumn{2}{|l|}{ Dislikes } \\
\hline It would be good to linkup with central records system & 2 \\
\hline I have to learn how to use the application & 3 \\
\hline 3D pain drawings would be useful & 1 \\
\hline Graphs for different people to be displayed simultaneously & 1 \\
\hline
\end{tabular}

A summary of the responses to this survey is given in Table II. This revealed that all the doctors appreciated the potential of the application in the prescription of treatment, given the close link between medication and pain location and type. Moreover, three of the surveyed clinicians viewed the observation of pain across time as useful in itself, but especially as regards the monitoring of the effect of any analgesics prescribed to the patient. On the downside (it is to be remarked that not all clinicians interviewed gave a full list of three "dislikes"), the prevalent concerns expressed were those of coming to grips with the functionality of the application, and of integrating it with the central IT repository. While the application is currently 

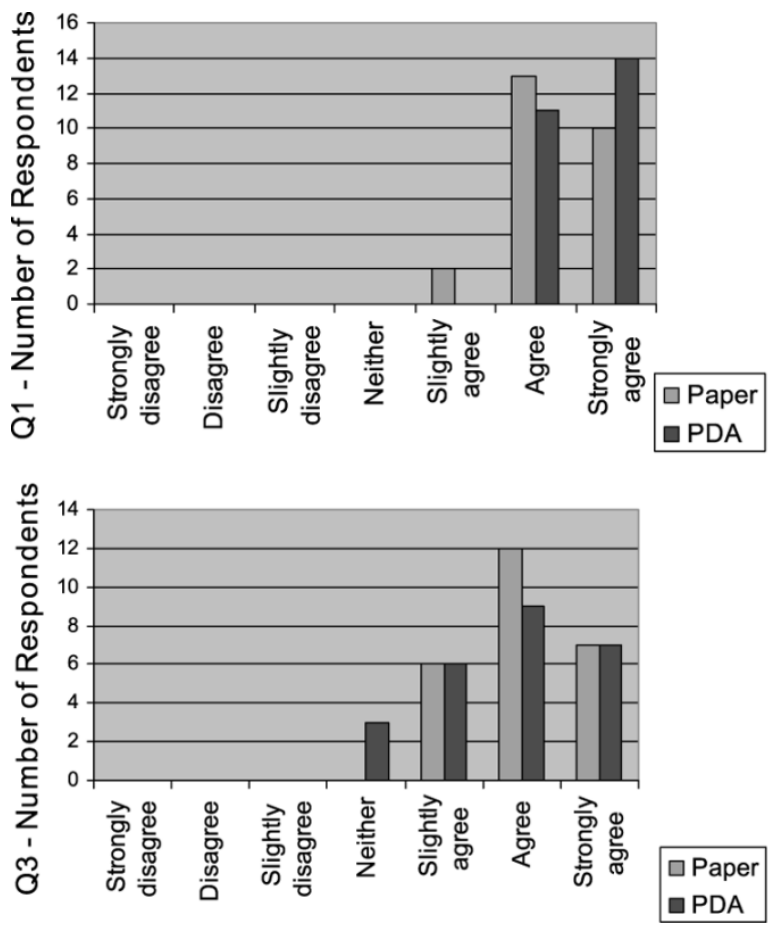

Fig. 12. Histograms of responses to evaluation questions.

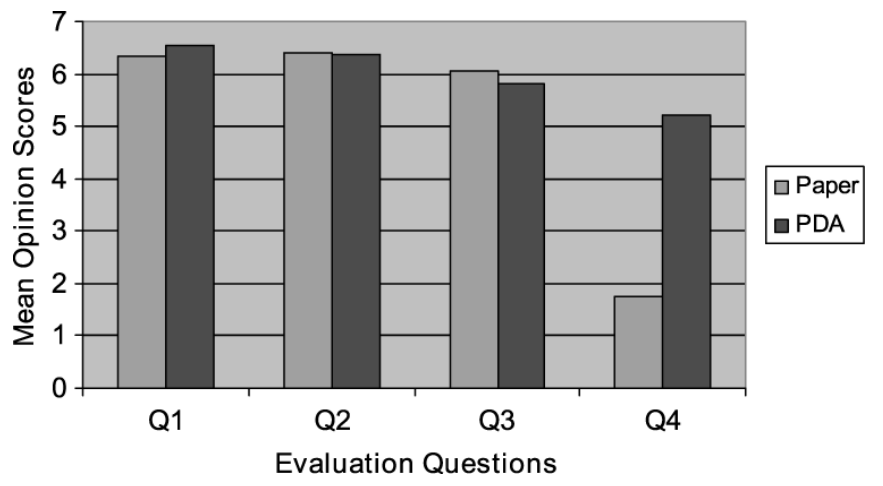

Fig. 13. Evaluation results: Paper-based approach versus PDA.

at a prototypical stage, the latter suggestion could indeed be implemented in future versions.

\section{CONCLUSION}

The challenge of pervasive clinical data collection has only now become attainable through recent advances in mobile and wireless communication capabilities. Only by harnessing the combined features and capacities of such technologies will healthcare applications be able to offer stakeholders location-independent communications and computing.

In this paper, we have described the design, implementation, and evaluation of a wireless-enabled solution for back-pain data collection and wireless transmission to a remote clinical database. Employing a user-friendly visual approach to data input, in our solution, such activities are carried out in an ubiquitous fashion, on a PDA; the fact that the collected data, including pain drawings, are digitized makes it easier to collect and analyze, while the fact that such input takes place on a PDA means that this can happen irrespective of the location of the user, and
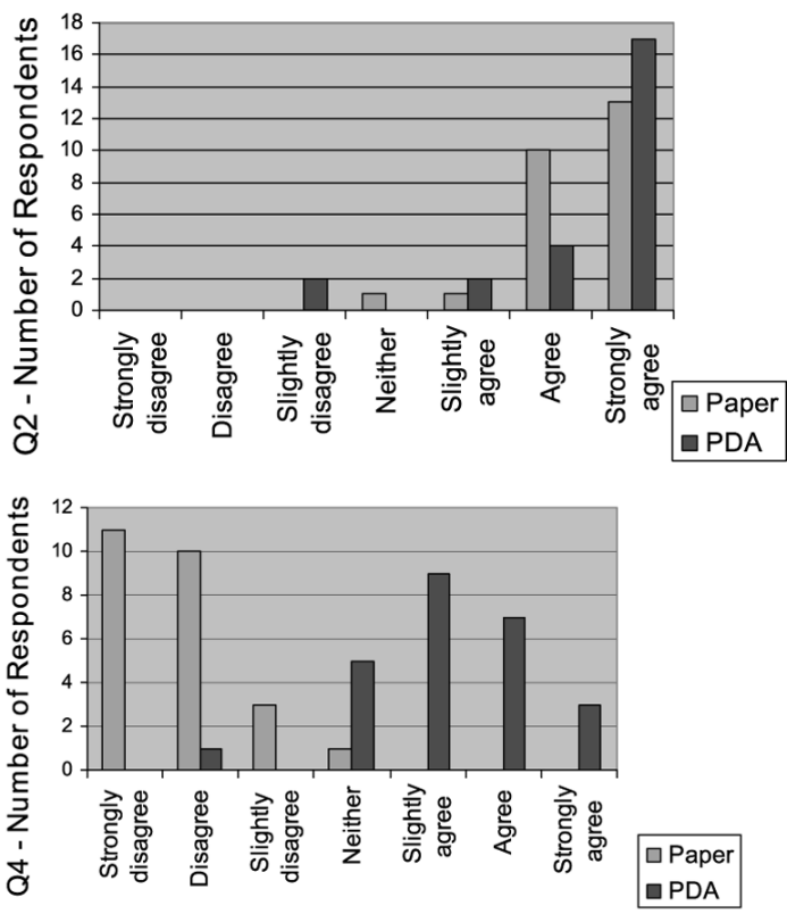

can be easily done at different times of the day. Moreover, our approach also enables the easy recording of time-sensitive information, with no clinical supervision. Finally, recognising the mobility problems that many back-pain patients endure, our solution is WiFi-enabled, thus facilitating remote, ubiquitous, data access and management, and absolving patients of the need to actually physically hand in their completed questionnaires.

We recognize, however, that our study has exposed certain limitations with current technologies, especially when these are handled by differently-abled individuals. Our proof-of-concept study has not addressed issues such as scalability, and security will need to be updated in line with future developments. Nonetheless, our experience has shown that the provision of such goals are worth pursuing, for only then will the potential of true anytime/anywhere data collection become be realized.

\section{REFERENCES}

[1] J. C. Lin, "Applying telecommunication technology to health-care delivery," IEEE Eng. Med. Biol. Mag., vol. 18, no. 4, pp. 28-31, Jul./Aug. 1999.

[2] O. Sheng et al., "Urban teleradiology in Hong Kong," J. Telemed. Telecare, vol. 3, no. 2, pp. 71-77, 1997.

[3] H. K. Huang, Basic Principles and Applications. New York: Wiley, 1999.

[4] M. Takizawa et al., "The mobile hospital-An experimental telemedicine system for the early detection of disease," J. Telemed. Telecare, vol. 4, no. 3, pp. 146-1551, 1998.

[5] R. W. Jones et al., "The AIDMAN project-Telemedicine approach to cardiology investigation, referral and outpatient care," J. Telemed. Telecare, vol. 6, pp. 32-34, 2000.

[6] R. J. Stone, "From engineering to surgery: the harsh reality of virtual reality," in Information Technologies in Medicine, M. Akay and A. Marsh, Eds. New York: Wiley, 2001, vol. 2, pp. 165-181.

[7] F. Magrabi, N. H. Lovell, and B. G. Celler, "Web based longitudinal ECG monitoring," in Proc. 20th Annu. Int. Conf. IEEE EMBS, vol. 20, 1998, pp. 1155-1158.

[8] S. Park et al., "Real-time monitoring of patient on remote sites," in Proc. 20th Annu. Int. Conf. IEEE EMBS, vol. 20, 1998, pp. 1321-1325. 
[9] B. Yang, S. Thee, and H. H. Asada, "A twenty-four hour tele-nursing system using a ring sensor," in Proc. IEEE Int. Conf. Robot. Autom., 1998, pp. 387-392.

[10] J. Grimson et al., "Sharing health-care records over the internet," IEEE Internet Comput., vol. 5, no. 3, pp. 49-58, May/Jun. 2001.

[11] T. Chan, "A web-enabled framework for smart card applications in health services," Commun. ACM, vol. 44, no. 9, pp. 77-82, 2000.

[12] M. E. Hernando et al., "Evaluation of DIABNET: A decision support system for therapy planning in gestational diabetes," Comput. Methods Programs Biomed., vol. 62, pp. 235-248, 2000.

[13] J. Huang et al., "An agent-based approach to health-care management," Appl. Artif. Intel., vol. 9, no. 4, pp. 401-420, 1995.

[14] A. J. López et al., "Web-based clinical decision support system using neural networks," in Proc. EUNITE 2002, Albufeira, Portugal, 2002, pp. $6-11$.

[15] A. V. Roudsari et al., "Web-based decision support and telemonitoring for the management of diabetes," in Proc. 22nd Annu. Int. Conf. IEEE Eng. Med. Biol. Soc., Chicago, IL, 2000, p. 1120.

[16] J. S. Hooda et al., "Health level-7 compliant clinical patient records system," in Proc. ACM 2004 Symp. Appl. Comput., Cyprus, 2004, pp. 259-263.

[17] M. Beyer et al., "Toward a flexible, process-oriented IT architecture for an integrated healthcare network," in Proc. ACM 2004 Symp. Appl. Comput., Cyprus, 2004, pp. 264-271.

[18] S. Ahn et al., "Embedded healthcare system based on SIP using telephone," in Proc. 6th Int. Conf. Adv. Commun. Technol., vol. 2, 2004, pp. 813-817.

[19] K. Hung and Y. Zhang, "Implementation of a WAP-based telemedicine system for patient monitoring," IEEE Trans. Inf. Technol. Biomed., vol. 7, no. 2, pp. 101-107, Jun. 2003.

[20] J. E. Bardram, "Applications of context-aware computing in hospital work: Examples and design principles," in Proc. ACM Symp. Appl. Comput., Cyprus, 2004, pp. 1574-1579.

[21] M. A. Munoz et al., "Context-aware mobile communication in hospitals," IEEE Computer, vol. 36, no. 9, pp. 38-46, Sep. 2003.

[22] N. J. Rodriguez et al., "PDA vs. Laptop: A comparison of two versions of a nursing documentation application," in Proc. 16th IEEE Symp. Computer-Based Med. Syst., 2003, pp. 201-206.

[23] J. Gibson and A. O. Frank, "Pain experienced by Electric Powered Indoor/Outdoor Chairs (EPIOC) users: A pilot exploration using pain drawings," in Proc. 2nd Meeting Eur. Fed. Phys. Rehab. Med., Vienna, Austria, May 2004, p. 114.

[24] A. Boucher. (1999) The prevalence of back pain in Great Britain in 1998. Dept. of Health. [Online]. Available: http://www.doh.gov.uk/public/back pain.htm

[25] A. Frank and L. H. De Souza, "Conservative management of low back pain," Int. J. Clin. Practice, vol. 55, no. 1, pp. 21-31, 2000.

[26] J. R. Jefferson and P. J. McGrath, "Back pain and peripheral joint pain in an industrial setting," Arch. Phys. Med. Rehab., vol. 77, pp. 385-390, 1996.

[27] F. Balague et al., "Non-specific low back pain in children and adolescents: Risk factors," Eur. Spine J., vol. 8, pp. 429-438, 1999.

[28] C. L. Von Baeyer et al., "Invalid use of pain drawings in psychological screening of back pain patients," Pain, vol. 16, pp. 103-107, 1983.

[29] B. M. Ginzburg et al., "The relationship between pain drawings and the psychological state," Pain, vol. 35, pp. 141-145, 1988.

[30] J. Hildebrandt et al., "The use of pain drawings in screening for psychological involvement in complaints of low-back pain," Spine, vol. 13, no. 6, pp. 681-685, 1988.
[31] N. H. Mann, III et al., "Initial-impression diagnosis using low back pain patient pain drawings," Spine, vol. 18, no. 1, pp. 41-53, 1992.

[32] H. Parker et al., "The use of the pain drawing as a screening measure to predict psychological distress in chronic low back pain," Spine, vol. 20, no. 2, pp. 236-243, 1995.

[33] A. O. Ransford et al., "The pain drawing as an aid to psychologic evaluation of patients with low-back pain," Spine, vol. 1, no. 2, pp. 127-134, 1976.

[34] A. Uden et al., "Pain drawings in chronic back pain," Spine, vol. 13, no. 4, pp. 389-392, 1988.

[35] H. Palmer, "Pain charts: A description of a technique whereby functional pain may be diagnosed from organic pain," New Zealand Med. J., vol. 48, no. 264, pp. 187-213, 1949.

[36] C. Ohlund et al., "Quantified pain drawing in subacute low back pain," Spine, vol. 21, no. 9, pp. 1021-1031, 1996.

[37] J. J. Rankine et al., "Pain drawings in the assessment of nerve root compression: A comparative study with lumbar spine magnetic resonance imaging," Spine, vol. 23, no. 15, pp. 1668-1676, 1998.

[38] C. W. Chan et al., "The pain drawing and Waddell's nonorganic physical signs in chronic low-back pain," Spine, vol. 18, no. 3, pp. 1717-1722, 1993.

[39] D. D. Ohnmeiss, "Repeatability of pain drawings in a low back pain population,” Spine, vol. 25, no. 8, pp. 980-988, 2000.

[40] R. J. Gatchel et al., "Qualifications of lumbar function: Part 6: The use of psychological measures in guiding physical function restoration," Spine, vol. 11, pp. 36-42, 1986.

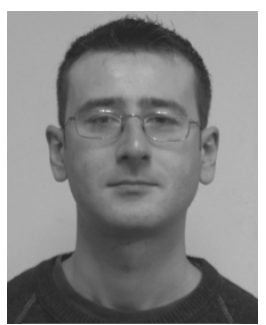

Tacha Serif received the B.Sc. (Hons) degree in information systems engineering in 2000, and the M.Phil. degree in computing in 2002, both from the University of Manchester Institute of Science and Technology (UMIST), Manchester, U.K.

$\mathrm{He}$ is a Ph.D. Researcher with the School of Information Systems, Computing and Mathematics, Brunel University, Uxbridge, U.K. His research interests include pervasive computing, mobile infotainment, wireless networks, and location-based and context-aware systems.

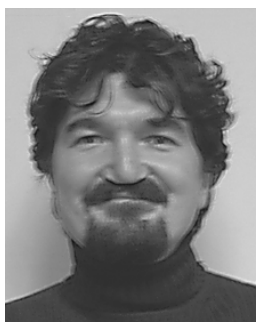

Gheorghita Ghinea (M'02) received the B.Sc. and B.Sc. (Hons) degrees in computer science and mathematics in 1993 and 1994, respectively, and the M.Sc. degree in computer science in 1996, from the University of the Witwatersrand, Johannesburg, South Africa, and the Ph.D. degree in computer science from the University of Reading, Reading, U.K., in 2000.

$\mathrm{He}$ is a Lecturer in the School of Information Systems, Computing and Mathematics, Brunel University, Uxbridge, U.K. His research interests pervasive computing, telemedicine, quality of service and multimedia resource allocation, and computer networking and security issues. 